\title{
Energy landscape of a Lennard-Jones liquid: Statistics of stationary points
}

\author{
Kurt Broderix*, Kamal K. Bhattacharya*, Andrea Cavagna ${ }^{\dagger}$, Annette Zippelius* and Irene Giardina** \\ * Institut für Theoretische Physik, Universität Göttingen, Bunsenstr. 9, D-37073 Göttingen, Germany \\ ${ }^{\dagger}$ Department of Physics and Astronomy, The University, Manchester, M13 9PL, United Kingdom \\ ** Service de Physique Thèorique, CEA Saclay, 91191 Gif-sur-Yvette, France
}

(November 1, 2018)

\begin{abstract}
Molecular dynamics simulations are used to generate an ensemble of saddles of the potential energy of a Lennard Jones liquid. Classifying all extrema by their potential energy $u$ and number of unstable directions $k$, a well defined relation $k(u)$ is revealed. The degree of instability of typical stationary points vanishes at a threshold potential energy $u_{t h}$, which lies above the energy of the lowest glassy minima of the system. The energies of the inherent states, as obtained by the StillingerWeber method, approach $u_{t h}$ at a temperature close to the mode-coupling transition temperature $T_{c}$.
\end{abstract}

The complex physical behaviour of supercooled liquids and glasses has always stimulated a description of such systems in terms of dynamical evolution upon a very complicated potential energy landscape [1]. In particular, the presence of many inequivalent glassy minima of the potential energy gives rise to a rich pattern of activated dynamics and has therefore attracted most of the attention of the community in the past years. As a consequence, the analysis of the structure of minima has become the main focus of the energy landscape approach to glasses. Based on the pioneering work of Goldstein [1] and of Stillinger and Weber [2], a number of authors have investigated the statistical properties of local minima, which are reached by a gradient descent, starting from an equilibrium configuration of the supercooled liquid state at temperature $T[3,6]$. The energies of the inherent minima sampled after the quench reveal two characteristic temperatures: At low $T$ the system gets trapped in a very small number of basins and falls out of equilibrium. Although this transition temperature depends on the cooling rate, it is very close to the dynamical transition $T_{c}$ of mode-coupling theory (MCT) [- A second higher temperature marks the onset of nonexponential relaxation, which has been associated with energy landscape dominated dynamics $[3$.

A method which does not focus purely on the properties of minima, is the instantaneous normal mode (INM) approach [8]: the spectrum of the eigenvalues of the Hessian matrix is computed and averaged over all the configurations with the Boltzmann distribution at temperature $T$. The INM analysis focuses on two points: First, it has been suggested that the barrier heights and hopping rates can be obtained from the INM spectrum [8]. Second, the temperature where the fraction $f(T)$ of negative eigenvalues of the INM spectrum goes to zero is interpreted as the point where the number of directions for free diffusion in phase space vanishes, thus giving an estimate of $T_{c}$, below which activation remains as the only mechanism of diffusion [9]. The INM analysis has been criticized [10], because equilibrium configurations with unstable directions are in general not stationary points of the potential energy, even if the force vanishes along the unstable directions [11.

In contrast to the INM method, which is an intrinsically thermal approach by sampling configurations according to their Boltzmann weight, we focus here on the purely geometric properties of the energy landscape. We investigate all the stationary points of the potential energy, be they minima, or unstable saddles [12]. We classify them according to their number of unstable directions, (index $\mathrm{K}$ ), their energy and the smallest eigenvalue of the Hessian matrix. Thereby we can address the following questions: Is there a threshold energy for saddles, such that for energies below this threshold, it is very unlikely to find saddles and the dynamic relaxation of the system requires activation? Is there a signature of the threshold energy in the dynamical behaviour of the system? What is the typical energy difference between saddles of index $K$ and $K+1$ and can this be taken as an estimate of the potential energy barriers?

The system under consideration is a binary mixture of large (L) and small (S) particles with $80 \%$ large and $20 \%$ small particles. Small and large particles only differ in diameter, but have the same mass. They interact via a Lennard-Jones potential of the form $V_{\alpha \beta}\left(r_{i j}\right)=$ $4 \epsilon_{\alpha \beta}\left[\left(\sigma_{\alpha \beta} / r_{i j}\right)^{12}-\left(\sigma_{\alpha \beta} / r_{i j}\right)^{6}\right]$, where $\vec{r}_{i}$ denotes the position of particle i $(i=1,2 \ldots N)$ and $r_{i j}=\left|\vec{r}_{i}-\vec{r}_{j}\right|$. All results are given in reduced units, where $\sigma_{L L}$ was used as the length unit and $\epsilon_{L L}$ as the energy unit. The other values of $\epsilon$ and $\sigma$ were chosen as follows: $\epsilon_{L S}=1.5, \sigma_{L S}=0.8, \epsilon_{S S}=0.5, \sigma_{S S}=0.88$ [13]. The systems were kept at a fixed density $\rho \approx 1.2$. Periodic boundary conditions have been applied and the potential has been truncated appropriately according to the minimum image rule [14]. We have applied a truncation procedure which on the one hand ensures the potential energy to be zero at the cut-off $r_{\text {cut }}$ and on the other hand provides a continuous first-derivative of $V_{\alpha \beta}(r)$ at $r_{\text {cut }}$. Throughout this study we present results for systems with $N=60$ particles using $r_{\text {cut }}=1.8$. A few samples with up to $N=120$ have been simulated to validate our results. 
In order to explore the geometric properties of the stationary points of the potential energy, we use the following method: We start by equilibrating a random initial configuration of $N$ particles at a given temperature $T$ using a standard molecular dynamics simulation technique. After equilibration the system evolves for a time $\tau_{\text {run }}$. To locate a saddle of the potential energy close to the equilibrated configuration we look for the absolute minima of the modulus square of the force. In order achieve this we perform a quench on a pseudo-potential energy landscape $W(x)$ given by $W(x)=\vec{\nabla} U(x) \cdot \vec{\nabla} U(x)$, whereas the original potential energy is defined by $U(x)=\sum_{1 \leq i<j \leq N} V_{\alpha \beta}\left(r_{i j}\right)$. Notice that all absolute minima of $W(x)$ are stationary points of $U(x)$, hence every saddle of $U(x)$ has a well defined basin of attraction. The local minima of $W(x)$ do not correspond to zeros of the real force. These points are frequently sampled; they can easily be distinguished from the absolute minima and are excluded from the following analysis.

Given a stationary point we consider the number of negative eigenvalues of its Hessian matrix, that is the index $K$ of the saddle. We compute the index density $k=K /(3 N)$ and the potential energy density $u=U / N$ of the saddle and plot these values in the $(u, k)$ plane. In Fig. 1 we show the results for many saddles, which were sampled with the steepest descent procedure on the pseudo-potential $W(x)$ for two different values of the temperature. This plot clearly suggests that there is an underlying curve $k(u)$, which is independent of temperature and which encodes a purely geometric feature of the landscape. By sampling stationary points at different values of $T$ we are exploring different regions of the potential energy surface and thus different portions of the same geometric curve $k(u)$.

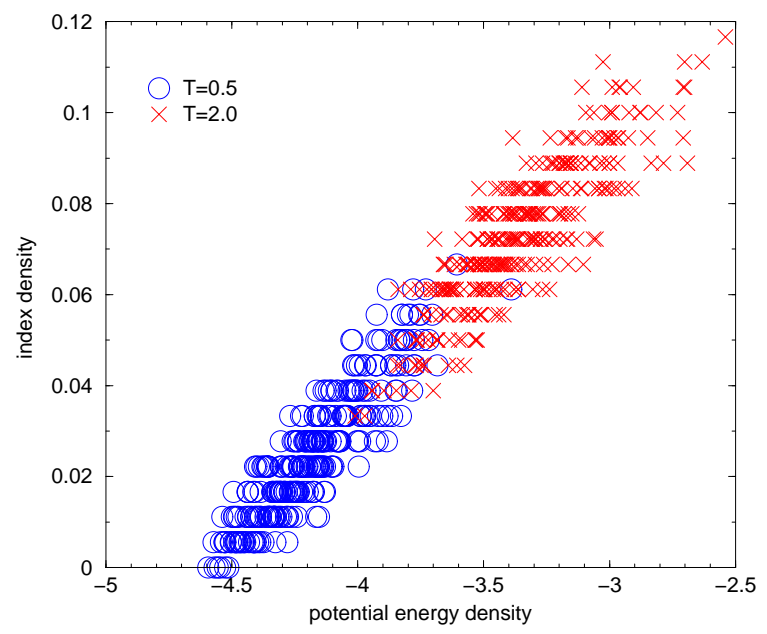

FIG. 1. Index density as a function of the potential energy density: sampling of stationary points at two different temperatures, $T=0.5$ and $T=2.0$.

To further support this conjecture we have sampled stationary points at various temperatures and have averaged all data in two different ways: Firstly, we have considered all the stationary points with a given index density and we have computed their average potential energy (this is possible because for a finite system $k$ can only assume discrete values). We call this procedure geometric average. Secondly, we consider all the stationary points obtained at a given temperature $T$ and we compute their average index $k(T)$ and energy $u(T)$. Eventually, we plot $k$ and $u$ parametrically in $T$ on the $(u, k)$ plane. We call this the parametric average. If our sampling of the saddles is a fair exploration of the underlying geometric space, then the two averages must coincide. This is what happens, as it is shown in Fig.2.

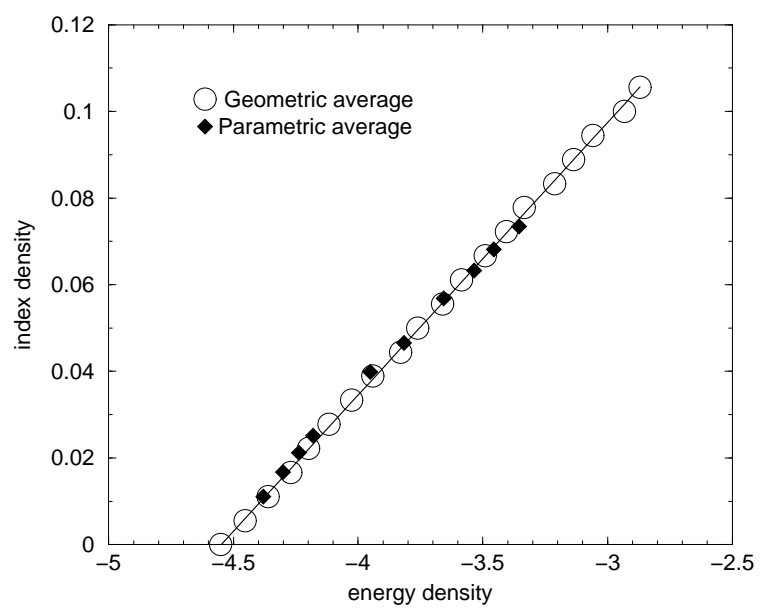

FIG. 2. Index density as a function of the potential energy density. Average over all the data obtained by sampling at $T \in[0.3,2.0]$. The full line is a linear fit of the geometric average.

The averaged data as plotted in Fig.2 reveal a unique function $k(u)$. In other words, if we cut the potential energy landscape with a plane of constant energy density $u=u_{0}$, the stationary points on this plane (or within a narrow shell around this plane) will be dominated by saddles with index density $k\left(u_{0}\right)$. Furthermore, $k(u)$ is to a very good approximation linear up to an index density of $10 \%$ negative eigenvalues. This implies that the curve extrapolates to zero at a well defined energy, which we call the threshold energy, $u_{t h}$, in analogy with spinglasses [15]. In Fig.3 we present a magnification of the last four points of $k(u)$, showing that the linear interpolation of all the data and the linear interpolation of the last four points give the same estimate for the threshold energy, that is $u_{t h}=-4.55$.

The threshold energy marks the border between the saddles-dominated portion of the energy landscape and the minima-dominated one. An interesting point is that $u_{t h}$ is above the energy of the lowest lying minima we find, $u_{0}=-4.65$, as shown in Fig.3 ( $u_{0}$ is obtained from an extensive search for minima of the potential energy using the Stillinger-Weber method). This implies the ex- 
istence of a finite energy density interval where minima are overwhelmingly more numerous than saddles. The same phenomenon has been observed in mean-field models of spin-glasses [15] exhibiting one step replica symmetry breaking (1RSB).

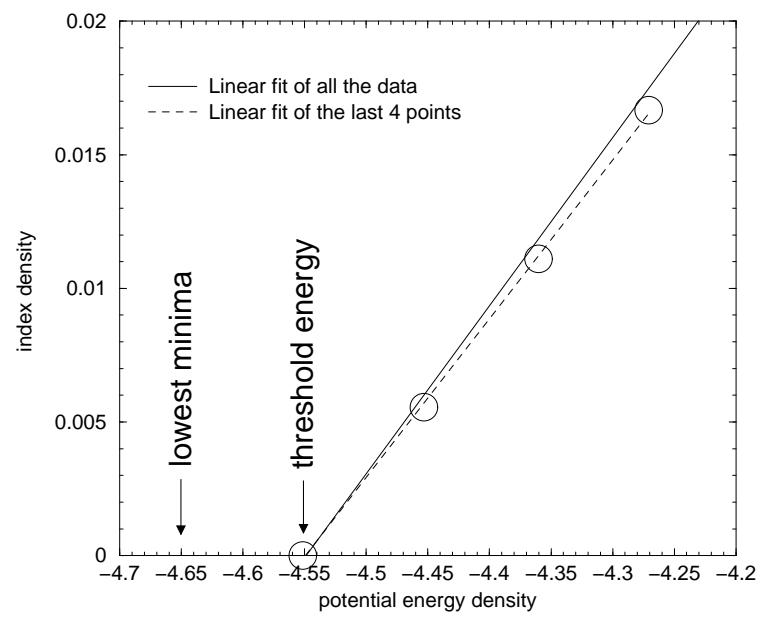

FIG. 3. Magnification of Fig.2. With the arrows are indicated the energy $u_{0}$ of the lowest minima found in the system and the threshold energy $u_{t h}$.

In Fig. 4 we plot the lowest eigenvalue $\lambda_{0}$ of the Hessian as a function of the energy density. As expected, $\lambda_{0} \rightarrow 0$ for $u \rightarrow u_{t h}$, implying that most extrema have a small number of eigenvalues close to zero. Somewhat surprising is the approximately linear dependence of $\lambda_{0}(u)$ on energy, $\lambda_{0} \sim\left(u_{t h}-u\right)$, exactly as in 1RSB spin-glasses [15].

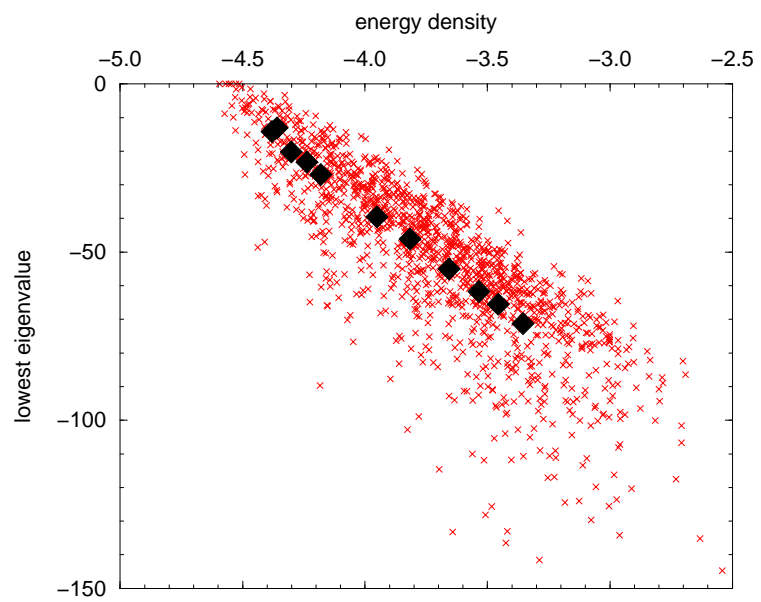

FIG. 4. Lowest eigenvalue of the Hessian as a function of the energy density. Full diamonds are the parametric average of all the data obtained at the same values of $T$ as in Fig.2.

It is important to relate the geometric features of the energy landscape to the dynamical behaviour of the supercooled liquid close to the glass transition. The index density vanishes at the threshold so that minima are the dominant stationary points below $u_{t h}$. We therefore expect to find a signature of $u_{t h}$ in the dynamics or, more specifically, a link between the threshold energy and the onset of activated dynamics upon cooling. To that end we have used our MD simulations at temperature $\mathrm{T}$ [3, 4 ] to compute the energy density $u_{\min }(T)$ of local minima reached by gradient descent on the potential energy surface. These data are compared in Fig.5 to the difference $\delta(T)=<U / N>(T)-3 T / 2$ of the average potential energy density $\langle U / N\rangle(T)$, as calculated in the MD simulation, and the vibrational energy in the harmonic approximation. For a harmonic potential $\delta(T)$ is just the energy of the minimum of the well. In Fig.5 we see that $\delta(T) \sim u_{\min }(T)$ for $T \leq 1.2$ [16]. This is the range of temperatures which is dominated by the energy landscape and the timescales for the two processes of relaxation - vibrations inside a minimum and hopping between different minima - start to separate. Close to the glass transition the system falls out of equilibrium, as indicated in Fig. 5 by the saturation of both quantities, $u_{\min }(T)$ and $\delta(T)$. The temperature where this happens is known to depend on the equilibration time of the MD simulation (see e.g. [3]).

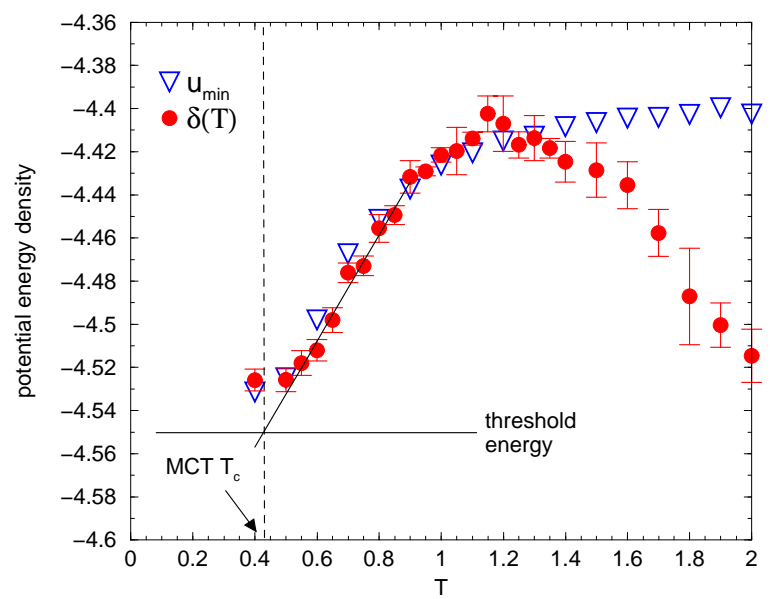

FIG. 5. Triangles represent the average energy of local minima $u_{\min }$ as a function of the temperature of the initial MD trajectory. Circles represent the quantity $\delta(T)=\langle U / N\rangle(T)-3 / 2 T$.

Extrapolating that part of the curve corresponding to temperatures where equilibration is ensured (see Fig. 5 ), one observes that both $u_{\min }(T)$ and $\delta(T)$ reach the threshold energy at a temperature approximately equal to the MCT transition $T_{c} \sim 0.44$ 13. The crossover from a non-activated dynamics above $T_{c}$ to an activated one below $T_{c}$ 17,6], may thus be interpreted in this context as a geometric transition from saddle dominated, to minima dominated regions of the potential energy landscape.

We can obtain an estimate of the energy barrier $\Delta U=$ $1 /\left(3 k^{\prime}\right) \sim 5.0$ from the slope $k^{\prime}(u)$ of the linear function [18]. This estimate has the right order of magnitude, see e.g. [6]. Note however that we do not know which saddles are accessible from a particular minimum via a dynamic 
trajectory. Furthermore we have not yet computed the entropic contribution to the transition rate, which is necessary for an estimate of the free energy barrier and hence for a comparison with the experimental time scales.

Finally in Fig.6, we compare our result for $k(u)$ to an INM analysis. For the latter we have computed the average fraction of negative eigenvalues for equilibrium configurations as a function of their energy (parametric average). We see that for all temperatures the INM index is higher than the index of genuine saddles at the corresponding energy. Hence the average curvature is overestimated by INM, as expected [10].

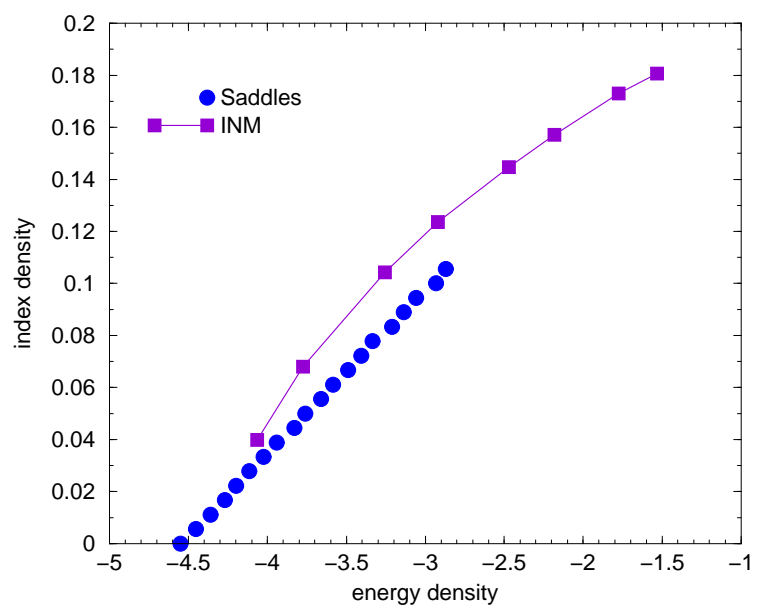

FIG. 6. INM vs saddle index density as functions of the potential energy density. $T \in[0.3,2.0]$.

In this Letter we have presented a new method to characterize in a quantitative way the geometric structure of the potential energy landscape, which goes beyond the analysis of local minima. We have shown that the typical saddles index $k$ depends linearly on the potential energy density and we have defined a threshold energy, $u_{t h}$, where the degree of instability vanishes. Furthermore, the threshold energy has been related to the MCT transition temperature $T_{c}$.

The method of steepest descent on the pseudopotential $W(x)$ is powerful enough to provide detailed information on the properties of saddles, so that one can estimate not only energy barriers but also the entropic contribution to the transition rates. Given that we know all eigenvalues of the Hessian at the stationary points, we can evaluate the partition function at the saddle, which is needed as an input for the transition state theory. So far we have mainly looked at one system size, i.e. 60 particles, and have only checked a few results for larger systems. It would be interesting to study the effects of varying system size on barrier energies, entropies and number of unstable directions. Another important point is the mutual accessibility of minima and saddles. This latter problem may be tractable by a combination of both, steepest descent on the potential energy and steep- est descent on the pseudo-potential surface. Work along these lines is in progress.

\section{ACKNOWLEDGMENTS}

A.C. thanks A. Bray, F. Colaiori, T. Keyes, M. Moore, G. Parisi, F. Ritort, S. Sastry and F. Thalmann for useful discussions. The work of A.C. was supported by EPSRC(UK) Grant GR/L97698 and the work of K.B. by the DFG under grant No. Br 1894/1-1.

In memory of Kurt.

[1] M. Goldstein, J. Chem. Phys 51, 3728 (1969).

[2] F.H. Stillinger and T.A. Weber, Phys. Rev. A 25, 978 (1982).

[3] S. Sastry, P.G. Debenedetti and F. Stillinger, Nature, 393, 554 (1998).

[4] K. K. Bhattacharya, K. Broderix, R. Kree and A. Zippelius, Europhys. Lett. 47, 449 (1999)

[5] W. Kob, F. Sciortino and P. Tartaglia, Europhys. Lett 49, 590 (2000)

[6] T.B. Schrøder, S. Sastry, J.C. Dyre and S. Glotzer, J. Chem. Phys. 112, 9834 (2000).

[7] U. Bengtzelius, W. Goetze and A. Sjolander, J. Phys. Chem. 17, 5915 (1984); E. Leutheusser, Phys. Rev. A 29 2765 (1984).

[8] T. Keyes, J. Chem. Phys. A 101, 2921 (1997). B. Madan and T. Keyes, J. Chem. Phys. 98, 3342 (1992).

[9] See, for example, S. Sastry, Phys. Rev. Lett. 76, 3738 (1996); F. Sciortino and P. Tartaglia, Phys. Rev. Lett. bf 78, 2385 (1997).

[10] J.D. Gezelter, E. Rabani and B.J. Berne, J. Chem. Phys. 107, 4618 (1997).

[11] S. Bembenek and B. Laird, Phys. Rev. Lett. 74, 936 (1995).

[12] For a similar analysis, see also L. Angelani, R. Di Leonardo, G. Ruocco, A. Scala and F. Sciortino, preprint cond-mat (17 July 2000).

[13] W. Kob and H. C. Andersen, Phys. Rev. Lett. 73, 1376 (1994); Phys. Rev. E 51, 4626 (1995)

[14] M.P. Allen and D.J. Tildesley, Computer Simulations of Liquids, Oxford Science Publications, Oxford (1996).

[15] J. Kurchan, G. Parisi and M.A. Virasoro, J. Phys. I France 3, 1819 (1993); L.F. Cugliandolo and J. Kurchan, Phys. Rev. Lett. 71, 173 (1993); A. Cavagna, I. Giardina and G. Parisi, Phys. Rev. B 57, 11251 (1998).

[16] For an equivalent investigation, see also S. Sastry, J. Phys.: Condens. Matter 12, 6515 (2000).

[17] C. A. Angell, J. Phys. Chem. Sol. 49, 863 (1988).

[18] A. Cavagna, Europhys. Lett. 53, 490 (2001); preprint cond-mat/9910244 (1999). 